\title{
A Corpus-Based Study on the Comparative Degree Errors in English Writing
}

\author{
Yongzhen $\mathrm{Pu}^{1}$, Huaqing $\mathrm{He}^{*^{2}}$ \\ ${ }^{1}$ Fushun County No.2 Middle School, Fushun, Sichuan, China \\ ${ }^{2}$ College of Foreign Language Education, China West Normal University, Nanchong, Sichuan, China \\ ${ }^{2}$ masha4567@sina.com
}

\begin{abstract}
Language learning is a process of trial and error, which indicates that language errors cannot be completely avoided in the course of learning English. Some scholars have noticed that language errors can be collected, analyzed and corrected, and scientific error analysis can provide language learners reduce or avoid those errors consciously. This thesis mainly applies error analysis method to discuss the comparative degree errors in ST2, and those errors are classified into seven groups: miss choosing analysis or synthesis; omitting comparative degree markers; duplicative use of analysis and synthesis; overuse comparative degree; comparative structure error; irregular adjective errors; the rest errors (unidentified errors).
\end{abstract}

Keywords: comparative degree, corpus, error analysis.

\section{INTRODUCTION}

Writing is considered as one of the most important purposes of learning English. Comparison, constantly used in writing, is a feature in the morphology or syntax of some languages, whereby adjectives and adverbs are inflected or modified to indicate the relative degree of the property defined by the adjective or adverb (McArthur, 1992). According to the change of adjective or adverb in comparative degree sentences, comparative degree can be divided into two categories: synthesis and analysis.

Although it is known that writing is important in English learning, some English researchers realize that the English writing ability of Chinese English learners is far less than satisfaction, and one of the reasons is language error. "Errors are the flawed side of learner speech and writing (Delay, 1982)." Some scholars have noticed that language errors can be collected, analyzed and corrected, and scientific error analysis can provide language learners with enlightenment to help students to reduce or avoid errors consciously.

ST2, a sub-corpus of CLEC (Chinese Learner English Corpus), is a collection of Chinese high students' written text. Without limitation on the writing topic and writing time, those compositions are written after class, containing about 200,000 words. Different errors in this corpus are marked with different symbols, and comparative degree error is marked with "aj3" which will be collected and analyzed in this thesis.

\section{LiteratURE REVIEW}

\subsection{Corpus-Based Error Analysis}

Corpus, a data bank mainly for research, consists of scientifically and organically collected language in authentic situations and the language can be written or spoken. Corpus is aimed at being a carrier to analyze language learners' acquisition, especially non-native language learners' acquisition. "Corpora can be considered as a type of foreign language writing aid as the contextualized grammatical 
knowledge acquired by non-native language users through exposure to authentic texts in corpora allows learners to grasp the manner of sentence formation in the target language, enabling effective writing." (Yoon \& Hirvela, 2004) With the development of technology, corpus allows "researchers not only to count categories in traditional approaches to language but also to observe categories and phenomena that have not been noticed before" (Hunston, 2002).

As a main tool for English language research, corpus has a diverse range of uses. For language teaching, corpus can give information about how a language works that may be accessible to native speaker intuition, such as the detailed phraseology. In addition, some language features can be proved by calculation which can provide quantitative data. For example, nearly all the future time references in conversational English are indicated by will or other models. Such information is important for syllabus and materials design.

\subsection{Studies on English Comparative Degree}

A large number of studies about English comparative degree have been carried out, and there are three mainstream studies about English comparative degree in foreign countries. The first one is carried out from the perspective of grammar. The second one is carried out from the perspective of sentence structure. The third one puts focus on the adjectives or adverbs that contain the mark of comparative degree.

There are four mainstreams about the comparative degree research at home. The first one is about its grammar. The second one is to compare the comparative degree difference between Chinese and English, such as, comparisons of comparative degree acquisition between the first language and the second language and comparisons of comparative degree's cognition. The third one is focusing on the adjectives. The fourth one aims to study the translation of comparative degree. This kind of study mainly summarized the key points of translation.

The results of studies on English comparative degree contain theoretic and practical meaning. However, most of these studies are qualitative studies, lacking of statistics or specific evidence to support their findings. In order to compensate for this disadvantage, this study focuses on analyzing the comparative degree errors in high students' writing.

\section{RESULTS AND DISCUSSION}

\subsection{Classification of Comparative Degree Errors}

This part aims at categorizing and analyzing the 48 comparative degree errors marked with "aj3" which have been retrieved in Antconc.

Although the errors expressions are different, some of them are basically the same in nature, which makes it possible and have sense to divide these errors into groups and then analyze the possible reasons by the means of reflection. "Aj3" errors are easily sorted out with the help of Concordance. Then according to their features in error, they are classified into seven groups.

According to table 4, there are four types of comparative degree errors, which appear about or more than 10 times, including group one duplicative use analysis and synthesis, group two omitting markers of comparative degree, group three mischoosing analysis and synthesis, and group five overuse comparative degree. The four groups take up about four in five of all the errors, which makes it meaningful and necessary to analyze these error groups in detail. 
Table1. Error Classification

\begin{tabular}{|c|c|c|c|}
\hline error type & examples & frequency & percentage \\
\hline mischoosing & More and more fast, more clever, activer, more long, etc & 14 & $29.2 \%$ \\
\hline omitting markers & $\begin{array}{l}\text { dirty than, beautiful and beautiful, much powerful than, } \\
\text { etc }\end{array}$ & 10 & $20.8 \%$ \\
\hline duplicative use & more cheaper, more better, more thicker, more wetter, etc & 9 & $18.8 \%$ \\
\hline Overuse & spent more time, very harder, get up late, etc & 8 & $16.7 \%$ \\
\hline $\begin{array}{l}\text { wrong sentence } \\
\text { structure }\end{array}$ & $\begin{array}{l}\text { teachers eagerer than, more important and more } \\
\text { important, etc }\end{array}$ & 3 & $6.3 \%$ \\
\hline $\begin{array}{l}\text { wrong irregular } \\
\text { adjectives }\end{array}$ & less exercises, farer & 2 & $4.2 \%$ \\
\hline Others & most colder, more beautiful colors in the world & 2 & $4.2 \%$ \\
\hline Total & & 48 & $\approx 100 \%$ \\
\hline
\end{tabular}

Mischoosing analysis and synthesis takes up the highest percentage with $29.2 \%$, nearly one third of all comparative errors, including the following sentences:

1. They're the basicest [aj3,1-] food in our life.

2. But [wd4,1-2] Cuba team is one of the excellentest [aj3,1-1] team in the world a

3. The Emperor was even fooler [wd2,s-][aj3,4-5] to walk in the porcession [fm1,-]

4. People's living standard became good [aj3,4-5][wd3,4-3] more and more[sn9,-]

5. because the time of the match was more and more fast [aj3,10-0]

6. It's $[\mathrm{wd} 3,-8]$ computing speed is more fast [aj3,5-3]than a man. [sn8,s]

7. I am taller and more heavy [aj3,5-3] than last year. but the eyesight is worse than last year.

8. wrote down the awkwardest [aj3,1-1] position [cc3,6-6] in the history of my class.

9. We [fm3,-] were do [vp6,1-2] much activer [aj3,1-]. the [fm3,-] money box were

10. you are the most great [aj3,s-] person in my heart.

11. many places of interest the [fm3,-] famousest [aj3, s-] one of them is [np7,s-] Great Wall.

12. This time, I attend[wd3,-] to running of the 400 mertres. It is more [aj3,0-1] long to run.

13. But the old professor was more clever [aj3,1-0] than the two impostors.

14. To children, the most happy [aj3,2-0] day in a year is the Spring Festival.

All of these errors result from choosing wrong comparative structure, especially about the form of adjectives or adverbs. For those monosyllabic adjectives which should adopt suffix "er" to form comparative degree, students choose the modifier "more". For instance, fast is a monosyllabic adjective, so it ought to be added "er" to express comparative degree. On the contrary, for those polysyllabic adjectives which should be in the form of "more", students choose suffix "er" to form comparative degree form. The most confusing adjective is disyllable, because there is no absolute rule for them to choose analysis or synthesis form. As a matter of fact, most of disyllables should adopt "more", and only a small number of disyllables ought to adopt "er". Therefore, what we could do is to accumulate those commonly used disyllables, paying some attention to their comparative degree form, such as, active-more active, clever-cleverer, etc. However, some scholars and researchers hold the 
view that the range of "more" and "the most" application becomes wider and wider. Not only can it be applied to polysyllabic adjectives and most disyllabic adjectives, but also can be applied to some commonly used monosyllabic adjectives. (Cheng Shanzhen, 1988) Although this trend is accepted by some English learners or speakers, it is still not right in Chinese high school English learning, which indicates in order to get higher score in English examination, such case should be avoid.

The second top group is omitting markers of comparative degree, which takes up one in five of all the comparative errors, including the following sentences:

15. and wear new clothes. The children are [aj3,3-0] happiest.

16. too many people $[\mathrm{sn} 8, \mathrm{~s}]$ and the weather was not hot[aj3,-4] in the morning than afternoon

17. we hoped our teachers can [vp9,3-3] [wd4,3-3] beautiful and beautiful [aj3,3-]

18. At the age of seventeen, it [wd5,1-4] is the beautiful [aj3,s-] age of [wd3,4-] every one.

19. I hoped my little cousin would get sensible and sensible [aj3,9-0].

20. But it is not dirty [aj3,4-3] than last year.

21. I like his old songs much [aj3,3-4] than his new songs.

22. He couldn't see anything but he came back and said it [pr1,s-s] was most beautiful [aj3,3-0].

23. The China [wd2,-1] team and made the team much powerful [aj3,-1] than before

24. In China the southern part have [vp3,s-] much [aj3,s-] water than the northern part.

There are two types of omitting, one of which is omitting the marker of analysis or synthesis. For example, "hot in the morning than" omits the comparative degree marker "er". In fact, "hot" ought to be changed into "hotter". The other type is omitting the article "the" which only appears in superlative degree. "It was most beautiful" omits the definite article "the", so it should be corrected as "it was the most beautiful".

The third top group is duplicative use of analysis and synthesis, taking up about $18.8 \%$, including the following sentences:

25. When you go to work. [sn2,-] and [fm3,-s] radio is much more cheaper [aj3,2-] than television.

26. I hoped it was more better [aj3,1-] and better.

27. And the weather is hoter [fm2,-], the ice is more thicker [aj3,2-] [sn8,s].

28. The footprint was more larger [aj3,1-] for him.

29. The wet clothes absorb the water, ... more wetter [aj3,1-0].

30. Today, it's more colder [aj3,2-0].

31. Now, I'm growing up [vp6,s-], but she is more and more older [aj3,6-].

32. It is more [aj3,0-1] stronger and lively than before.

33. Computer, and new computer is more smaller [aj3,1-0] than the first one.

Although this comparative degree error type is simple, still some students cannot avoid it. As the rules of comparative degree settled, this is completely unacceptable. Those error expressions, such as "more cheaper", "more better", "more thicker" and so on should adopt synthesis form, and only keep "er".

The forth top group is the overuse of comparative degree, taking up $16.7 \%$, including the following 
sentences:

34. They were much warm [aj3,3-0], and said something.

35. If you notice it [pr3,3-0], you will get much happy[aj3,-]!

36. Some [fm3,-] maths problems were so difficult that I spent more [aj3,2-6] time

37. The school is not only bigger [aj3,5-3] but also beautiful.

38. The student of [pp1,2-6] having the match are very harder [aj3,10-0][wd3,s-]

39. They will play deep $[\mathrm{wd} 3,1-3]$ into the night, get up later [aj3,2-3] in next morning.

40. I have done more [aj3,3-2] English excerses [fm1,-], but I still lazy to [vp2,3-1] read and recide[fm1,-] the text, I am too lazy, how can I get rid of it [pr2, 22-0] ?

41. So I had a better [aj3,4-1] result.

Judging from the context, there is no intention or no need to apply comparative degree while students use it. The most distinct one is this sentence: the school is not only bigger but also beautiful. Looking through the whole passage, it can be figured out that the author just wanted to state the fact that his or her new school is big and beautiful, and there is no other schools used to compare with the school, thus it is quite apparent that comparative degree marker of "bigger" should be deleted. This type of error sometimes is not easy to be noticed, for context is the most essential factor that decides whether comparative is necessary in the sentence.

Wrong sentence structure only appears 3 times, but it also should be paid attention to and learners should try to avoid making this error. This group consists of the following sentences:

42. Sometimes our teachers [wd4,3-3] eagerer [aj3,3-2] than us.

43. English becomes more important and more important [aj3,7-].

44. Knowledge which the students taught [vp7,-] is dry [aj3,-2] as dust, and needed mechanical

Actually, this type of error can be divided into two categories. One is about the structure of the sentence, while the other one is about the structure of comparative degree. For example, "our teacher eagerer than us", this sentence contains two errors. The first one is lacking of verb, and the other one is mischoosing analysis or synthesis of comparative degree. As a result, a verb "are" should be added to this sentence, and "eagerer" should be corrected as "more eager".

Wrong irregular adjective error group includes the following sentences:

45. Oh, if the place is farer [aj3,3-2] than there.

46. I was so lazy that I did less [aj3,7-11] exersices [fm1,-]for my study.

Irregular adjectives refer to those adjectives whose comparative degree forms and superlative degree form have no regulation. Actually, irregular adjective is also an important part in comparative degree grammar. Despite this kind of error only appears twice in the corpus, it is also worth noticing, because the author has found that there are always some students make such errors in their homework. It is known to us that most English comparative adjectives obey to rules of analysis and synthesis, but there are some adjectives not consisting with the rules, so these adjectives have to be memorized particularly.

The rest two aj3 error sentences have nothing in common, so they belong to the last group, including the following sentences: 
47. It is most colder [aj3,3-2] than yesterday, but we are very happy.

48. Weave clothes of the more [aj3,s-] beautiful colors and designs [cc3,11-3] in the world,

\subsection{Causes for the Errors}

By the means of reflective thinking and error analysis, this part is going to discuss the possible reasons which may explain those comparative degree errors. The error analysis method gives feedback on learning effect of second language acquisition. It can provide language learners and teachers with specific clue or suggestion which aids to avoid the same type error. Reflective thinking is adopted to work out the real reasons, explaining why high school students make these errors.

With the opportunity interning in Fushun2 middle school, compositions text with comparative degree expression writing are easily collected. Class 16 grade one has 62 students. There are 59 pieces of compositions with title of "changes in senior high school". Then those comparative degree errors are deliberately selected out as a bridge to discover possible reasons for comparative degree errors in the corpus. Based on the error classification in 4.3, these comparative degree errors are classified into different groups. There are four groups of comparative degree error committed both in the composition written by my students and the compositions in ST2. On the basis of error classification discussed and reflective thinking from students, related reasons are figured out as followings.

\subsubsection{Interlingual Transfer}

Language transfer is "the influence results from the similarities and differences between the TL (target language) and any other language that has been previously acquired (Odlin, 1989)." Generally speaking, there are two kinds of transfer, positive transfer and negative transfer. Many empirical studies have proved that the interference of mother tongue is widely exists in the process of second language acquisition. (Ellis, 1981: 29) Corder has pointed out that when learners have trouble in expressing their idea with second language, they tend to appeal to their mother language. (Corder, 1978) As a result, for Chinese English learners, whether they use Chinese expression to compensate English unconsciously or purposely use Chinese expression as a learning strategy can easily lead to negative transfer of mother tongue. Actually, this kind of influence on English writing is a common phenomenon among Chinese senior high school students, so negative transfer of mother tongue has been studied by many researchers. It is reported that about 51\% English language errors result from the interference of mother language. (Ellis, 1999)

According to the reflective thinking of students, group of duplicative use comparative degree errors made by Chinese high school students greatly result from the influence of mother language, in other words, interlingual transfer has a great effect on the second language acquisition. Among all these comparative degree errors, duplicative use of analysis and synthesis, overuse may result from negative transfer of mother tongue.

In the corpus, there are 9 duplicative use errors. As we all know, English comparative degree are composed mainly by "adj-er" and "more adj", while Chinese comparative degree has nothing to do with the adjective, for it usually adds a word “更” or “越” before the adjective. This may easily confuse some English learners when they translate Chinese comparative degree into English, because they often translate all Chinese comparative markers into "more". For example, "more and more fast" may miswritten with the thought that “越来越快” are translated verbatim as “more and more fast".

\subsubsection{Language Learning Strategy}

"Language learning strategy is a term referring to the processes and actions that are consciously deployed by language learners to help them to learn or use a language more effectively." (Health, 2015) 
They have also been defined as "thoughts and actions, consciously chosen and operationalized by language learners, to assist them in carrying out a multiplicity of tasks from the very outset of learning to the most advanced levels of target language performance"(Andrew, 2011). Some language learners develop language rules while learning and using language. For example, in order to reduce language learning burden, some students tend to overuse or overgeneralization language rules. When they have leant two or more than two languages, they may choose those easy to remember and easy to use rules. Consequently, some errors come out as the result of the influence of improper language learning strategy.

Generalization is one of learning strategies. Language learners prefer to apply what they have learned previously to a new situation. However, this strategy can be easily overused, and then overgeneralization appears. Overgeneralization is a false learning strategy in which learners make generalizations based on a single piece of language phenomenon, i.e. it means the use of previously available language rules in new situation which is not the same condition with the previous one. Actually, overgeneralization belongs to intralingual transfer.

The two error groups: wrong irregular adjectives, mischoosing, have something to do with overgeneralization. For instance, "farer" is an clear overgeneralization example. The writer does know one of the rules of comparative degree, which is to add "er" to an adjective. However, it is wrong, because "far" is an irregular adjective, and its comparative degree deformation should be "farther" or "further". It is same to "more long". The writer knows one of the rules of comparative degree deformation, which is to add "more" before to an adjective, thus no matter it is monosyllable or polysyllable, he use the rule of adding "more".

\subsubsection{Incomplete Application of Rules}

Because of the incomplete knowledge of target language, learners do not master one language rule completely, especially a complicated language grammar, and they do not know how to use the rule exactly. As a result, some language errors are committed.

On the basis of analyzing the reflective thinking, especially from Wang Haoyu, incomplete application of rules is one of reasons accounting for these errors. There are two errors groups: omitting markers and wrong sentence structure have connection with this reason. For example, "dirty than" is an incomplete comparative degree structure. The learner does not know the rule and structure of comparative degree comprehensively. Therefore, the structure is not complete. This is the same to wrong sentence structure group.

Actually, the two groups of errors also, to some degree, due to the carefulness less. It is not difficult to find this reason in their reflective thinking. Five students admit that they do know the rule and structure of comparative degree, but when they are writing, they sometimes omit some part of sentence structure.

On the basis of previous study and the reflective thinking, only three reasons accounting for the comparative degree errors are discussed in this thesis, but there must be some other reasons which haven't been discovered.

\section{Conclusion}

Comparative degree is part of English grammars which cannot be ignored by English learners. Some Chinese high students think that comparative degree is rather simple, not paying much attention. As a matter of fact, it is not rare to find some comparative degree errors in the writing of Chinese high school students. This thesis applies Antconc to retrieve aj 3 which is the marker of comparative degree error in the corpus ST2. Firstly, all these errors are classified into different groups. Next, reflective 
thinking helps to reveal reasons for those errors.

According to the study, there are seven comparative degree error groups in this study. Group one is mischoosing analysis or synthesis, i.e., those adjectives which should adopt "er/est" add "more/the most", and vice verse, taking up 29.2\%. Group two is omitting comparative degree markers, i.e., there is no comparative degree marker "er/est" or "more/the most" in the sentences, taking up $20.8 \%$. Group three is duplicative use of analysis and synthesis, i.e., both "er" and "more" are added to comparative degree structure, taking up $18.8 \%$. Group four is overuse comparative degree, in other word, comparative degree applied to express idea while there is no context for comparative degree expression, taking up $16.7 \%$. Group five is wrong in comparative degree structure, such as: more important and more important, taking up 6.3\%. Group six is about irregular adjective errors, such as: farer, taking up $4.2 \%$. And the rest errors belong to group seven.

Three reasons accounting for those comparative errors are put forward in this thesis: interlingual transfer, learning strategies and incomplete application of rules.

Although some useful information has gained in this thesis through error analysis method and reflective thinking, there are still some disadvantages in this study. The frequency of comparative degree is not completely counted, because there are other comparative degree structures didn't retrieved, such as: less than, the same as, etc. Besides, in real language expressions, not all the sentences with comparative degree structure express comparative meaning, on the contrary, some sentences without comparative degree structures do have the meaning of comparison. Therefore, it is a complicated work to select out all the comparative sentences. As a result, there can and should be some further studies on this topic.

\section{REFERENCES}

Cohen, Andrew. 2011. Strategies in Learning and Using A Second Language [M](2nd ed.). Longman. Corder, S. P. 1978. Language-learner Language[J]. In J. C. Richards (Ed), Understanding Second and Foreign Language Learning[C]. Rowley, MA: Newbury House.

Delay, H. 1982. Language Two[M]. Oxford: Oxford University Press.

Ellis R. 1999. Understanding Second Language Acquisition[M], Shanghai: Shanghai Foreign Language Education Press.

Ellis, H. 1981. The Transfer of Learning[M]. Oxford: Oxford University Press.

Huddleston, R. \& G. K. Pullum. 2002. The Cambridge Grammar of English Language[M]. Cambridge: Cambridge University Press.

Rose. Heath. 2015. Researching language learning strategies. In Paltridge, Brian; Phakiti, Aek. Research methods in applied linguistics. Bloomsbury.

Terence Odlin. 1989. Language Transfer: Cross-Linguistic Influence in Language Learning [M], Cambridge University Press.

Tom McArthur. 1992. The Oxford Companion to the English Language [M], Oxford University Press. Yoon, H, \& Hirvela, A. ESL. 2004. Student Attitudes toward Corpus Use in L2 Writing. Journal of Second Language Writing, Elsevier Press, 257-283.

成善祯. 1988. 《现代英语中比权等级的若干发展变化》 [J], 上海外国语学院学报, (1).

\section{AUTHORS' BIOGRAPHY}

Corresponding Author: He Huaqing, female, who was born in Wanzhou, Chongqing city of China in 1970, is an English teacher in China West Normal University. The main area research is Applied Linguistics.

Pu Yongzhen, female, is an English teacher in Fushun County No.2 Middle School. The main area research is English teaching. 